\title{
Single-incision laparoscopic cholecystectomy
}

\author{
K. Thompson - A. Spivack $\cdot$ L. Fischer • \\ B. Wong $\cdot$ G. Jacobsen $\cdot$ M. Talamini • \\ S. Horgan
}

Received: 28 August 2009/Accepted: 10 November 2009/Published online: 11 November 2010

(C) The Author(s) 2010. This article is published with open access at Springerlink.com

\begin{abstract}
Background Single-incision laparoscopic surgery (SILS) has been proposed as a minimally invasive technique with the advantages of smaller external scars and reduced pain. Furthermore, usage of the flexible endoscope for SILS in lieu of the standard laparoscope provides distinct visualization advantages. This video shows a single-incision cholecystectomy performed using a single incision placed through the umbilicus.

Methods A 39-year-old woman with chronic symptomatic cholelithiasis was enrolled under institutional review board protocol to undergo SILS. She had previously undergone a laparoscopic tubal ligation. A single incision was made using the previous umbilical incision, and the abdomen was entered in an open fashion. The flexible endoscope was placed directly through the fascial incision, with two 5-mm ports on either side. Adhesions to the gallbladder were taken down with the harmonic scalpel. Dissection proceeded using an articulating grasper and retraction to identify the cystic duct and artery. The duct and artery were serially clipped and divided. The cystic duct was additionally secured with a loop ligature. The gallbladder was cauterized from the liver bed using the articulating hook cautery and extracted through the wound.
\end{abstract}

Presented at the SAGES 2009 Annual Meeting, April 22-24, 2009, Phoenix, AZ.

Electronic supplementary material The online version of this article (doi:10.1007/s00464-010-1445-8) contains supplementary material, which is available to authorized users.

K. Thompson $(\bowtie) \cdot$ A. Spivack · L. Fischer · B. Wong ·

G. Jacobsen · M. Talamini - S. Horgan

University of California-San Diego, San Diego, CA, USA

e-mail: kjthompson@ucsd.edu
Results The final incision placed at the base of the umbilicus was $7 \mathrm{~mm}$ long. The operative time was $58 \mathrm{~min}$, with minimal blood loss recorded. The patient was discharged home on the day of the procedure and did not experience any postoperative complications.

Conclusions Single-incision cholecystectomy can be performed safely through one incision in the umbilicus, optimizing cosmesis. Substitution of the flexible endoscope for the standard laparoscope allows many greater degrees of visualization in SILS. This allows clear identification of the biliary ductal anatomy, allowing cholecystectomy to proceed safely. Placement of the endoscope directly through the incision decreases the profile of ports through the incision and increases maneuverability.

Keywords Cholecystectomy · Endoscopy · Single-incision laparoscopy

Disclosures K. Thompson, A. Spivack, L. Fischer, B. Wong, G. Jacobsen, M. Talamini, and S. Horgan have no conflicts of interest or financial ties to disclose.

Open Access This article is distributed under the terms of the Creative Commons Attribution Noncommercial License which permits any noncommercial use, distribution, and reproduction in any medium, provided the original author(s) and source are credited. 